\title{
Effect and Safety of Meropenem-Vaborbactam versus Best-Available Therapy in Patients with Carbapenem-Resistant Enterobacteriaceae Infections: The TANGO II Randomized Clinical Trial
}

\author{
Richard G. Wunderink · Evangelos J. Giamarellos-Bourboulis · Galia Rahav • Amy J. Mathers • \\ Matteo Bassetti · Jose Vazquez • Oliver A. Cornely · Joseph Solomkin • Tanaya Bhowmick • \\ Jihad Bishara · George L. Daikos • Tim Felton • Maria Jose Lopez Furst • Eun Jeong Kwak • \\ Francesco Menichetti · Ilana Oren · Elizabeth L. Alexander · David Griffith · Olga Lomovskaya • \\ Jeffery Loutit $\cdot$ Shu Zhang $\cdot$ Michael N. Dudley $\cdot$ Keith S. Kaye
}

Received: August 13, 2018 / Published online: October 1, 2018

(c) The Author(s) 2018

\section{ABSTRACT}

Introduction: Treatment options for carbapenem-resistant Enterobacteriaceae (CRE) infections are limited and CRE infections remain

Enhanced digital features To view enhanced digital features for this article go to https://doi.org/10.6084/ m9.figshare.7083281.

Electronic supplementary material The online version of this article (https://doi.org/10.1007/s40121018-0214-1) contains supplementary material, which is available to authorized users.

R. G. Wunderink ( $\square)$

Division of Pulmonary and Critical Care,

Department of Medicine, Feinberg School of

Medicine, Northwestern University, Chicago, IL,

USA

e-mail: r-wunderink@northwestern.edu

E. J. Giamarellos-Bourboulis

4th Department of Internal Medicine, National and Kapodistrian University of Athens, Medical School, Athens, Greece

G. Rahav

Infectious Disease Unit and Laboratories, Sheba Medical Center and Sackler Faculty of Medicine, Tel Aviv University, Tel Aviv, Israel

\section{A. J. Mathers}

Infectious Diseases and International Health, Department of Medicine, University of Virginia

School of Medicine, Charlottesville, VA, USA associated with high clinical failure and mortality rates, particularly in vulnerable patient populations. A Phase 3, multinational, openlabel, randomized controlled trial (TANGO II) was conducted from 2014 to 2017 to evaluate the efficacy/safety of meropenem-vaborbactam monotherapy versus best available therapy (BAT) for CRE.

Methods: A total of 77 patients with confirmed/suspected CRE infection (bacteremia, hospital-acquired/ventilator-associated bacterial pneumonia, complicated intra-abdominal infection, complicated urinary tract infection/

\section{Bassetti}

Infectious Diseases Clinic, Department of Medicine, University of Udine and Azienda Sanitaria Universitaria Integrata di Udine, Udine, Italy

\section{J. Vazquez}

Division of Infectious Diseases, Medical College of Georgia/Augusta University, Augusta, GA, USA

O. A. Cornely

Cologne Excellence Cluster on Cellular Stress Responses in Aging-Associated Diseases (CECAD), Zentrum für klinische Studien (ZKS Köln), and Department I of Internal Medicine, University Hospital of Cologne, Cologne, Germany

\section{J. Solomkin}

Department of Surgery, University of Cincinnati

College of Medicine, Cincinnati, OH, USA 
acute pyelonephritis) were randomized, and 47 with confirmed CRE infection formed the primary analysis population (microbiologic-CREmodified intent-to-treat, mCRE-MITT). Eligible patients were randomized 2:1 to meropenem-vaborbactam $(2 \mathrm{~g} / 2 \mathrm{~g}$ over $3 \mathrm{~h}$, q8h for 7-14 days) or BAT (mono/combination therapy with polymyxins, carbapenems, aminoglycosides, tigecycline; or ceftazidimeavibactam alone). Efficacy endpoints included clinical cure, Day-28 all-cause mortality, microbiologic cure, and overall success (clinical cure + microbiologic eradication). Safety endpoints included adverse events (AEs) and laboratory findings.

Results: Within the mCRE-MITT population, cure rates were $65.6 \%(21 / 32)$ and $33.3 \%(5 / 15)$ [95\% confidence interval (CI) of difference, $3.3 \%$ to $61.3 \% ; P=0.03)]$ at End of Treatment and 59.4\% (19/32) and 26.7\% (4/15) (95\% CI of difference, $4.6 \%$ to $60.8 \% ; P=0.02$ ) at Test of Cure;.Day-28 all-cause mortality was $15.6 \%$ (5/32) and 33.3\% (5/15) (95\% CI of difference, $-44.7 \%$ to $9.3 \%$ ) for meropenem-vaborbactam

\footnotetext{
T. Bhowmick

Division of Allergy, Immunology, and Infectious Diseases, Department of Medicine, Rutgers Robert Wood Johnson Medical School, New Brunswick, NJ, USA

J. Bishara

Infectious Disease Unit, Rabin Medical Center, Beilinson Hospital and Sackler Faculty of Medicine, Tel Aviv University, Tel Aviv, Israel

G. L. Daikos

First Department of Medicine, National and Kapodistrian University of Athens, Laiko Hospital, Athens, Greece

T. Felton

University Hospital of South Manchester and Division of Infection, Immunity and Respiratory Medicine, University of Manchester, Manchester, UK

M. J. L. Furst

Unidad de Infectología, Sanatorio Municipal Dr.

Julio Méndez, Buenos Aires, Argentina

E. J. Kwak

Division of Infectious Diseases, Department of Medicine, University of Pittsburgh Medical Center, Pittsburgh, PA, USA
}

versus BAT, respectively. Treatment-related AEs and renal-related AEs were $24.0 \%(12 / 50)$ and $4.0 \%(2 / 50)$ for meropenem-vaborbactam versus $44.0 \%(11 / 25)$ and $24.0 \%(6 / 25)$ for BAT. Exploratory risk-benefit analyses of composite clinical failure or nephrotoxicity favored meropenem-vaborbactam versus BAT $(31.3 \%$ [10/32] versus $80.0 \%$ [12/15]; $95 \%$ CI of difference, $-74.6 \%$ to $-22.9 \% ; P<0.001)$.

Conclusions: Monotherapy

with meropenem-vaborbactam for CRE infection was associated with increased clinical cure, decreased mortality, and reduced nephrotoxicity compared with BAT.

Clinical Trials Registration: NCT02168946.

Funding: The Medicines Company.

Keywords: Best available therapy; Carbapenemresistant Enterobacteriaceae; Meropenemvaborbactam; Randomized clinical trial; TANGO II

\section{F. Menichetti}

Dipartimento di gastroenterologia e malattie infettive, Azienda Ospedaliero-Universitaria Pisana, Pisa, Italy

\section{Oren}

Infectious Diseases Unit, Rambam Health Care

Campus, Haifa, Israel

E. L. Alexander · S. Zhang

The Medicines Company, Parsippany, NJ, USA

D. Griffith · O. Lomovskaya · J. Loutit .

M. N. Dudley

The Medicines Company, San Diego, CA, USA

K. S. Kaye

Professor of Internal Medicine, Director of Clinical Research, Division of Infectious Diseases, University of Michigan Medical School, 5510A MSRB I, SPC 5680, 1150 W. Medical Center Drive, Ann Arbor, MI 48109-5680, USA

e-mail: keithka@med.umich.edu 


\section{INTRODUCTION}

Increasing antimicrobial resistance, particularly among Gram-negative pathogens, has resulted in a critical need for new antibiotics [1]. Government agencies and public health authorities have initiated programs and policies for the development of new antibiotics for these pathogens, in large part due to the spread of beta-lactam resistance [2-5]. Ten million deaths per year by 2050 are estimated if antimicrobial resistance is not adequately addressed [6].

Carbapenems have been a last defense against extended-spectrum beta-lactamase-resistant pathogens [7]. Dissemination of carbapenemases [e.g., Klebsiella pneumoniae carbapenemase (KPC)] among Enterobacteriaceae has reduced the activity of carbapenems and other agents for these pathogens [8]. Consequently, treatment options for carbapenemresistant Enterobacteriaceae (CRE) infections are limited [9-11] and CRE infections remain associated with high clinical failure and mortality rates, particularly in vulnerable patient populations [12-16].

Vaborbactam is a first-in-class, boron-based, beta-lactamase inhibitor, with activity against serine beta-lactamases, especially optimized for potent KPC inhibition. Combination with meropenem restores activity against KPC-producing CRE in vitro and preclinical models [17]; this combination has a similar pharmacokinetic profile as meropenem alone [18].

The Targeting Antibiotic Non-susceptible Gram-negative Organisms (TANGO) I trial established the safety and effectiveness of meropenem-vaborbactam compared to piperacillin-tazobactam in complicated urinary tract infections (cUTI), including acute pyelonephritis (AP) $[19,20]$. VABOMERE was FDA-approved in the United States in 2017. TANGO II, a Phase 3 randomized trial, was conducted to evaluate the efficacy and safety of meropenem-vaborbactam monotherapy versus best available therapy (BAT) in adults with serious infections due to CRE.

\section{METHODS}

\section{Study Design and Participants}

TANGO II was a Phase 3, randomized, prospective, multicenter, multinational, open-label, active-controlled trial of adults with infections due to confirmed/suspected CRE. The trial enrolled patients from 27 hospital sites in 8 countries (Argentina, Brazil, Colombia, Greece, Israel, Italy, United Kingdom, United States) with known prevalence of KPC-producing CRE between November 2014 and June 2017. Protocol and informed consent form were approved by the sites' Institutional Review Boards/Independent Ethics Committees. The trial was conducted in accordance with the International Conference on Harmonisation Good Clinical Practice Guideline and with the 1964 Helsinki declaration and its later amendments or comparable ethical standards. Prior to initiation of study-related procedures, an informed consent form was signed by the patient or guardian/legal representative.

Prior to initiation of study-related procedures, an informed consent form was signed by the patient or guardian/legal representative. An independent Data Safety Monitoring Board (DSMB) reviewed accumulated safety data at scheduled intervals and serious adverse events on an ongoing basis. Once 72 patients were included in the safety and efficacy analysis, the DSMB determined the study met its stated objectives and advised discontinuing the study in its current form, as the risk/benefit analysis did not support ongoing randomization of patients to the BAT arm and would not be in the best interest of patients.

Eligible patients were age $\geq 18$ years with cUTI/AP, hospital-acquired/ventilator-associated bacterial pneumonia (HABP/VABP), bacteremia, or complicated intra-abdominal infection (cIAI), and confirmed or suspected (evidence in culture or molecular testing within past 90 days) CRE pathogen, requiring $\geq 7$ days of intravenous (IV) therapy (eTable 1). Patients with confirmed CRE infection were eligible if the baseline CRE pathogen was not susceptible to the current antimicrobial therapy (or the 
patient was not on antimicrobial therapy). If susceptible, patients were eligible if they had received $\leq 24 \mathrm{~h}$ of therapy before enrollment or had clinical deterioration/failure to improve after $\geq 48 \mathrm{~h}$ of therapy. Patients with suspected CRE infection who received $\leq 24 \mathrm{~h}$ of empirical Gram-negative antimicrobial therapy before enrollment were also eligible.

Key exclusion criteria included: history of significant hypersensitivity to beta-lactam antibiotics; confirmed infection with CRE-producing New Delhi metallo (NDM)-, Verona integron-encoded metallo-, imipenemase-metallo-, or oxacillinase-encoded beta-lactamases (based on local microbiology laboratory results); Acute Physiology and Chronic Health Evaluation II [21] score $>30$; or immediately life-threatening disease. Patients with impaired renal function, including hemodialysis, were eligible; those receiving continuous renal replacement therapy were not. Immunocompromised patients-leukemia or lymphoma (not in remission), prior solid organ/stem cell transplantation, neutropenia, or active receipt of immunosuppressive medications (including high-dose systemic steroids for $\geq 2$ weeks)—were eligible (NCT02168946).

\section{Bacterial Isolates}

CRE isolates were defined as Enterobacteriaceae with susceptibility results intermediate or resistant to carbapenems using Clinical and Laboratory Standards Institute (CLSI) 2014 criteria [22]. Carbapenem resistance was defined as a meropenem minimal inhibitory concentration $(\mathrm{MIC})>1 \mathrm{mg} / \mathrm{L}$ (or disk diffusion zone diameter $<23 \mathrm{~mm}$ ). If meropenem was not used for susceptibility testing, an isolate was considered carbapenem-resistant if intermediate or resistant to all carbapenems tested [22]. The causative pathogen had to be isolated from blood or infection site (urine, respiratory secretions, intra-abdominal fluid) cultures collected $\leq 3$ days before enrollment. Additional adequacy criteria for baseline pathogens differed by infection type (NCT02168946).

Additional infection-site specimens, when possible, and blood cultures were obtained immediately before the first dose of study drug. If unavailable or negative, the screening sample was used as the baseline sample. Isolates were sent to a central laboratory (JMI) for confirmatory culture, identification, and susceptibility testing using CLSI and European Committee on Antimicrobial Susceptibility Testing (EUCAST) criteria [23]. Central laboratory results were used for all analyses when available; if unavailable, local laboratory results were used (NCT02168946). Local microbiology laboratory results for culture and susceptibility testing were utilized for patient treatment purposes.

\section{Randomization and Blinding}

Eligible patients were randomized 2:1 to meropenem-vaborbactam or BAT using a computer-generated central randomization code and interactive voice/web response system. Randomization was stratified by infection type (cUTI/AP, HABP, VABP, cIAI, bacteremia) and region (North America, Europe, Asia Pacific, rest of world). Principal investigators and staff were not blinded to treatment assignment. A blinded local investigator also assessed clinical outcomes, and a blinded adjudication committee ensured unbiased assessments. The blinded adjudication committee decision was used for disagreement between the local blinded and unblinded investigator's evaluations.

\section{Interventions}

Patients randomized to meropenem-vaborbactam received 7-14 days of treatment as monotherapy (2-2 g) via IV infusion over $3 \mathrm{~h}$ every $8 \mathrm{~h}$. BAT included any of the following as monotherapy or in combination: polymyxins, carbapenems, aminoglycosides, or tigecycline; or monotherapy with ceftazidime-avibactam. Use of an aminoglycoside beyond $72 \mathrm{~h}$ in subjects with a pathogen(s) susceptible to meropenem-vaborbactam or ceftazidime-avibactam was considered a treatment failure. BAT was selected by the primary service and confirmed by the unblinded investigator according to institutional standards of care, patient characteristics (i.e., renal function, previous treatments, infection type, organism with corresponding MICs, etc.), and local regulatory approval. The choice of BAT regimen 
was left up to the investigator. Planned BAT was documented prior to randomization.

For patients with moderate-to-severe renal impairment (estimated creatinine clearance < $50 \mathrm{~mL} / \mathrm{min}$ ), meropenem-vaborbactam dose modifications were made (NCT02168946). BAT doses were adjusted according to local protocols.

\section{Outcomes and Assessments}

Efficacy endpoints across all infection types included: proportion of patients with clinical cure at the End of Treatment (EOT) and Test of Cure (TOC) $(7 \pm 2$ days after EOT); proportion of patients with microbiologic cure at EOT and TOC; and Day-28 all-cause mortality in the microbiologic-CRE-modified intent-to-treat (mCRE-MITT) population.

Primary efficacy endpoints for each infection type were based on FDA guidelines in the mCRE-MITT population and included: the proportion of patients that achieved overall success (composite endpoint of clinical cure + microbiologic eradication) at TOC in the cUTI/AP subgroup; all-cause mortality in the combined HABP/VABP and bacteremia subgroups; and the proportion of patients with clinical cure at TOC in the cIAI subgroup. Overall success at EOT was also assessed in the cUTI/AP subgroup. Secondary endpoints are listed in NCT02168946.

Clinical cure was defined as complete resolution of signs/symptoms of the index infection such that no further antimicrobial therapy (and/ or surgical intervention for cIAI) was warranted. Microbiologic cure was defined as microbiologic eradication or presumed eradication (clinical cure in absence of sample for repeat culture) at EOT and TOC (NCT02168946).

Treatment-emergent adverse events (TEAEs) and laboratory parameters were collected. AEs were coded using the Medical Dictionary for Regulatory Activities (v.17.0) and graded for severity according to the National Cancer Institute Common Terminology Criteria for Adverse Events [24]. Renal function was categorized according to changes in serum creatinine using Risk, Injury, Failure, Loss, or EndStage (RIFLE) criteria and change from baseline serum creatinine [25]. Clinical status (vital signs, physical exam) was assessed at baseline, Day 3, Day 7, EOT, TOC, and Late Follow-up, and as clinically indicated.

\section{Statistical Analysis}

\section{Analysis Populations}

Safety and MITT were the same and defined as all patients who received $\geq 1$ dose of study drug. Microbiologic modified intent-to-treat (m-MITT) populations included those who received $\geq 1$ dose of study drug and had a baseline qualifying Gram-negative pathogen. The primary study population was the mCREMITT population, defined as patients who received $\geq 1$ dose of study drug and had a baseline qualifying isolate confirmed as CRE by local or central laboratory (NCT02168946).

\section{Descriptive and Inferential Analysis Methods}

This was a descriptive study of patients infected with CRE pathogens; no formal power or sample size calculations were performed. However, in light of the magnitude and consistency of differences in clinical outcomes and all-cause mortality across patients in the primary population, ad hoc inferential testing was performed for selected outcomes. Differences in clinical cure at EOT and TOC and Day-28 all-cause mortality across all infection types were analyzed using the Wald test of equality. For exploratory analyses of composite endpoints of clinical failure or nephrotoxicity ( 2 definitions: either post-baseline increase in serum creatinine $\geq 1.0 \mathrm{mg} / \mathrm{dL}$; or $\mathrm{AE}$ preferred term of renal failure, renal failure acute, or renal impairment) and Day-28 all-cause mortality or nephrotoxicity (same definitions), Wald test of equality was used. For all study endpoints and analyses, descriptive summaries of proportion of patients in each group, and difference in proportions are provided (Table 1).

\section{RESULTS}

\section{Patient Disposition}

Seventy-seven patients were randomized: 52 to meropenem-vaborbactam and 25 to BAT 
Table 1 Baseline demographic and clinical characteristics (mCRE-MITT)

\begin{tabular}{|c|c|c|c|}
\hline Characteristic & $\mathrm{M}-\mathrm{V}(n=32)$ & BAT $(n=15)$ & Total $(N=47)$ \\
\hline Age, mean (SD), years & $63.5(14.1)$ & $60.2(13.0)$ & $62.5(13.7)$ \\
\hline \multicolumn{4}{|l|}{ Age cohort, $n(\%)$} \\
\hline$<65$ y & $17(53.1)$ & $9(60.0)$ & $26(55.3)$ \\
\hline$\geq 65 \mathrm{y}$ & $8(25.0)$ & $3(20.0)$ & $11(23.4)$ \\
\hline$\geq 75 y$ & $7(21.9)$ & $3(20.0)$ & $10(21.3)$ \\
\hline Female gender, $n(\%)$ & $18(56.3)$ & $5(33.3)$ & $23(48.9)$ \\
\hline White race, $n$ (\%) & $28(87.5)$ & $12(80.0)$ & $40(85.1)$ \\
\hline \multicolumn{4}{|l|}{ Region, $n$ (\%) } \\
\hline North America & $7(21.9)$ & $7(46.7)$ & $14(29.8)$ \\
\hline Europe & $19(59.4)$ & $8(53.3)$ & $27(57.4)$ \\
\hline Rest of World ${ }^{a}$ & $6(18.8)$ & $0(0)$ & $6(12.8)$ \\
\hline BMI, mean $(\mathrm{SD})$ & $27.9(9.0)$ & $25.8(7.6)$ & $27.2(8.5)$ \\
\hline \multicolumn{4}{|l|}{ Infection type, $n(\%)$} \\
\hline Bacteremia & $14(43.8)$ & $8(53.3)$ & $22(46.8)$ \\
\hline cUTI/AP & $12(37.5)$ & $4(26.7)$ & $16(34.0)$ \\
\hline HABP/VABP & $4(12.5)$ & $1(6.7)$ & $5(10.6)$ \\
\hline cIAI & $2(6.3)$ & $2(13.3)$ & $4(8.5)$ \\
\hline \multicolumn{4}{|l|}{ Baseline pathogen, $n(\%)^{\mathrm{b}}$} \\
\hline Klebsiella pneumoniae & $29(90.6)$ & $12(80.0)$ & $41(87.2)$ \\
\hline Escherichia coli & $3(9.4)$ & $1(6.7)$ & $4(8.5)$ \\
\hline Enterobacter cloacae sp. & $1(3.1)$ & $2(13.3)$ & $3(6.4)$ \\
\hline Proteus mirabilis & $0(0)$ & $2(13.3)$ & $2(4.3)$ \\
\hline Serratia marcescens & $1(3.1)$ & $1(6.7)$ & $2(4.3)$ \\
\hline Enrolled as confirmed CRE, $n(\%)$ & $23(71.9)$ & $14(93.3)$ & $37(78.7)$ \\
\hline Enrolled as suspected CRE, $n$ (\%) & $9(28.1)$ & $1(6.7)$ & $10(21.3)$ \\
\hline \multicolumn{4}{|l|}{ Creatinine clearance, $\mathrm{mL} / \mathrm{min}, n(\%)$} \\
\hline$\geq 50$ & $24(75.0)$ & $9(60.0)$ & $33(70.2)$ \\
\hline $30-49$ & $4(12.5)$ & $2(13.3)$ & $6(12.8)$ \\
\hline $20-29$ & $1(3.1)$ & $2(13.3)$ & $3(6.4)$ \\
\hline$<20$ & $2(6.3)$ & $0(0)$ & $2(4.3)$ \\
\hline Missing & $1(3.1)$ & $2(13.3)$ & $3(6.4)$ \\
\hline \multicolumn{4}{|l|}{ Charlson comorbidity index, $n(\%)$} \\
\hline$\leq 2$ & $4(12.5)$ & $1(6.7)$ & $5(10.6)$ \\
\hline
\end{tabular}


Table 1 continued

\begin{tabular}{|c|c|c|c|}
\hline Characteristic & $\mathrm{M}-\mathrm{V}(n=32)$ & $\operatorname{BAT}(n=15)$ & Total $(N=47)$ \\
\hline $3-4$ & $3(9.4)$ & $2(13.4)$ & $5(10.6)$ \\
\hline 5 & $11(34.4)$ & $1(6.7)$ & $12(25.5)$ \\
\hline$\geq 6$ & $14(43.8)$ & $11(73.3)$ & $25(53.2)$ \\
\hline Diabetes mellitus, $n(\%)$ & $12(37.5)$ & $7(46.7)$ & $19(40.4)$ \\
\hline SIRS, $n(\%)$ & $15(46.9)$ & $6(40.0)$ & $21(44.7)$ \\
\hline ICU admission, $n(\%)$ & $5(15.6)$ & $3(20.0)$ & $8(17.0)$ \\
\hline Immunocompromised $^{\mathrm{c}}, n(\%)$ & $11(34.4)$ & $8(53.3)$ & $19(40.4)$ \\
\hline Prior antibiotic failure ${ }^{\mathrm{d}}, n(\%)$ & $9(28.1)$ & $0(0)$ & $9(19.1)$ \\
\hline
\end{tabular}

$B A T$ best available therapy, $B M I$ body mass index, $C I A I$ complicated intra-abdominal infection, $C R E$ carbapenem-resistant Enterobacteriaceae, $c U T I / A P$ complicated urinary tract infection/acute pyelonephritis, $H A B P / V A B P$ hospital-acquired bacterial pneumonia/ventilator-associated bacterial pneumonia, ICU intensive care unit, $m C R E-M I T T$ microbiologic carbapenem-resistant Enterobacteriaceae modified intent to treat, $M-V$ meropenem-vaborbactam, $S D$ standard deviation, SIRS systemic inflammatory response syndrome

a Israel, Latin America (Colombia, Brazil, Argentina)

b Baseline pathogens listed occurred in 2 or more patients

c Receipt of immunosuppressive medications or bone marrow ablative chemotherapy, underlying lymphoma or leukemia (not in remission), previous transplantation, splenectomy, or presence of neutropenia

d Clinical evidence of prior antimicrobial failure as ascertained by the study investigator at screening and randomization

(Fig. 1). Among these, 75 (50 meropenemvaborbactam; $25 \mathrm{BAT}$ ) received $\geq 1$ dose of study drug, comprising the MITT and safety population. Of the 75 patients, 54 (35 meropenem-vaborbactam; 19 BAT) had a qualifying baseline Gram-negative pathogen (m-MITT population). Among these, 47 (32 meropenem-vaborbactam; 15 BAT) had microbiologically confirmed CRE infection (mCREMITT population).

In the MITT population, the most common infection types were bacteremia $(36.0 \%, 27 / 75)$ and cUTI/AP $(45.3 \%, 34 / 75)$. Another 9.3\% (7/75) were HABP/VABP and 9.3\% (7/75) were cIAI (eTable 2). A greater proportion of patients with prior antibiotic failure were randomized to meropenem-vaborbactam (10/50) than BAT $(0 / 25)$. Baseline characteristics inferred a vulnerable patient population: $28.0 \%(21 / 75)$ had renal impairment, $73.3 \%(55 / 75)$ had a Charlson Comorbidity Index $\geq 5,42.7 \%(32 / 75)$ had systemic inflammatory response syndrome, and $32.0 \%(24 / 75)$ met study pre-defined criteria for immune compromise. Klebsiella pneumoniae was the most common pathogen [58.7\% (44/75)] (eTable 2). Baseline demographic and clinical characteristics in the mCRE-MITT population were like those of the MITT population (Table 2).

In the mCRE-MITT population, K. pneumoniae was again the most common pathogen $(87.2 \%$ [41/47]) and meropenem MICs for K. pneumoniae were similar across treatment groups $\left(\mathrm{MIC}_{50}\right.$ $32 \mu \mathrm{g} / \mathrm{mL}, \mathrm{MIC}_{\text {range }} \leq 0.03$ to $>64 \mu \mathrm{g} / \mathrm{mL}$ in the meropenem-vaborbactam group and $\mathrm{MIC}_{50}$ $>32 \mu \mathrm{g} / \mathrm{mL}, \mathrm{MIC}_{\text {range }}>8$ to $>64 \mu \mathrm{g} / \mathrm{mL}$ in the BAT group) (eTable 3). The meropenem $\mathrm{MIC}_{50}$ for KPC-producing K. pneumoniae was $64 \mu \mathrm{g} / \mathrm{mL}$ in both groups. Five K. pneumoniae isolates showed a meropenem-vaborbactam MIC $>4 \mu \mathrm{g} / \mathrm{mL}$ (3 patients randomized to meropenem-vaborbactam; 2 to BAT): 4 produced metallo-beta-lactamases or class D carbapenemases (NDM or OXA-48) and 1 produced KPC-3 (randomized to BAT). Among organisms cultured after randomization, $1 / 32(3.1 \%)$ in the meropenem-vaborbactam group developed $\mathrm{a} \geq 4$-fold increase in MIC to meropenem-vaborbactam during 


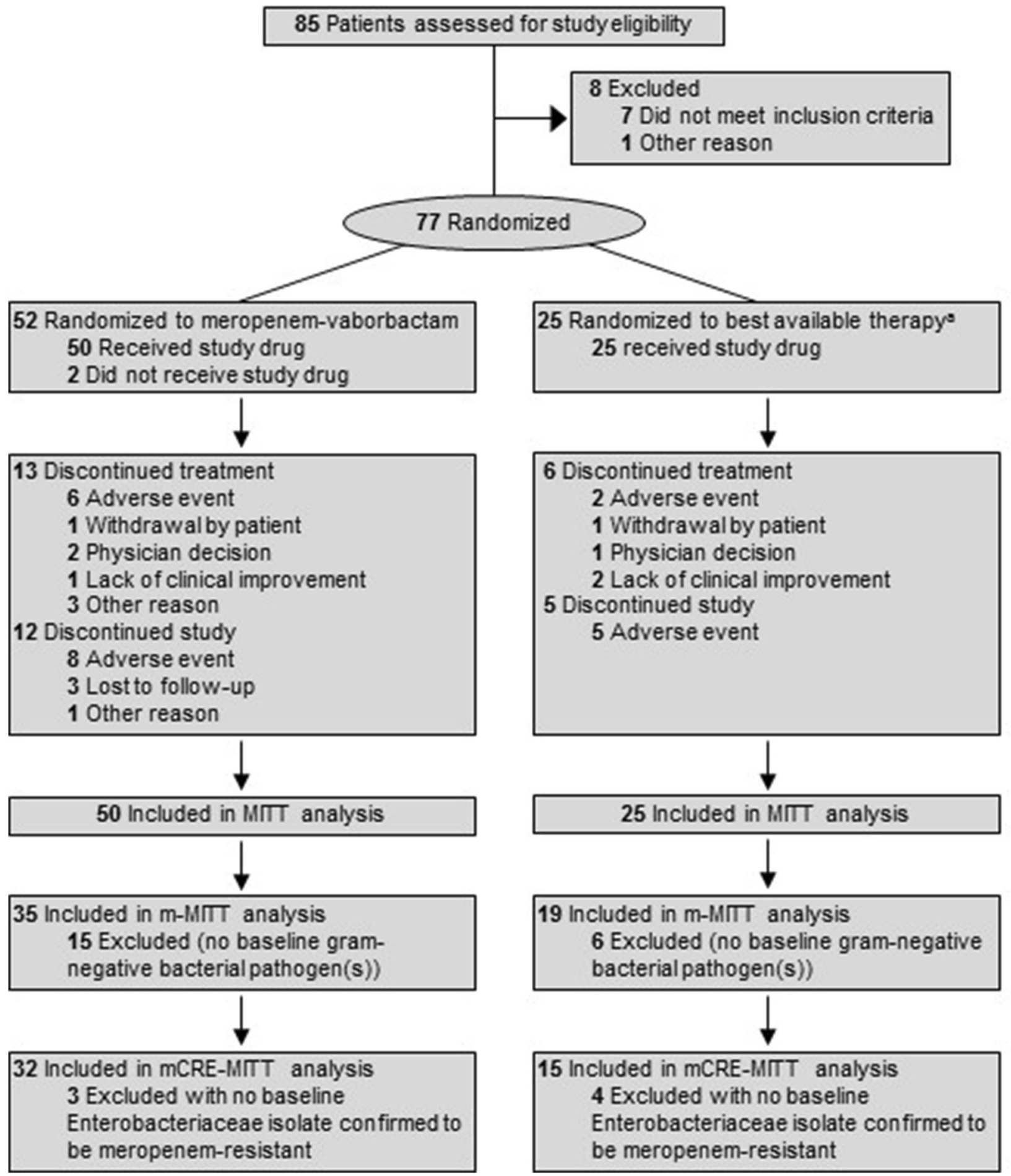

Fig. 1 Flow of patients in TANGO II. $m C R E-M I T T$ microbiologic-carbapenem-resistant Enterobacteriaceaemodified intent-to-treat, MITT modified intent-to-treat, $m$-MITT microbiologic modified intent-to-treat; $M-V$ meropenem-vaborbactam, $V A B P$ ventilator-associated

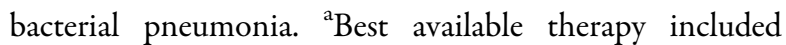
(alone or in combination): a carbapenem, aminoglycoside, polymyxin $\mathrm{B}$, colistin, tigecycline, or (monotherapy only) ceftazidime-avibactam 


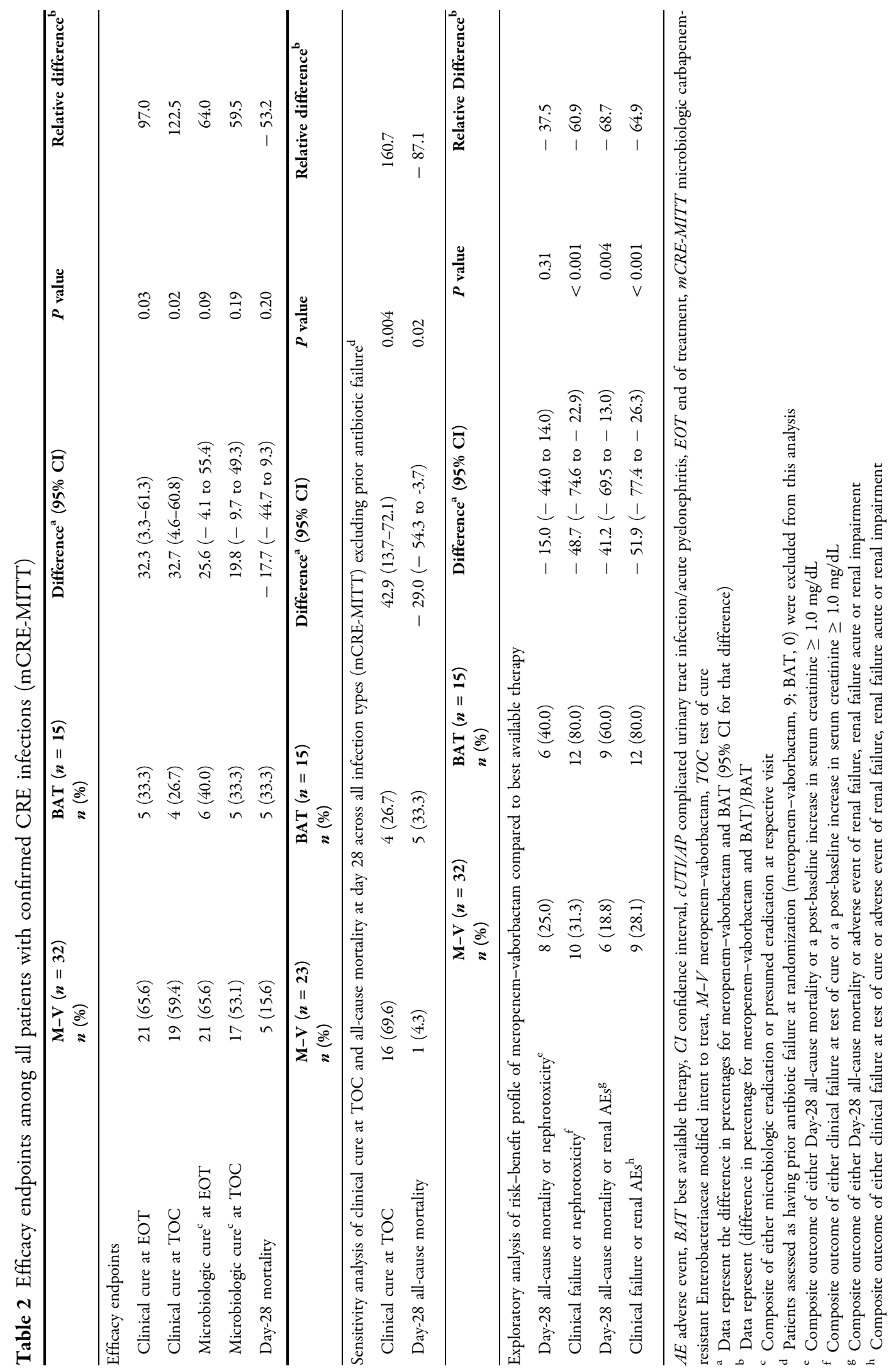


treatment (from 0.25 to $1 \mu \mathrm{g} / \mathrm{mL}$ ). A range of BAT treatments were given (eTables 4 and 5); 60.0\% of patients received at least one antimicrobial agent to which the baseline CRE pathogen was susceptible by CLSI breakpoints. In the BAT group, $1 / 15(6.7 \%)$ developed $a \geq 4$-fold increase in MIC to a BAT regimen (polymyxin + aminoglycoside, eTables 4 and 5): polymyxin, from 1 to $>4 \mu \mathrm{g} / \mathrm{mL}$; and gentamicin, from 1 to $>16 \mu \mathrm{g} / \mathrm{mL}$. The mean (standard deviation) duration of treatment exposure was 9.3 (3.9) days for meropenem-vaborbactam and 9.1 (4.0) days for BAT.

\section{Efficacy}

In the mCRE-MITT population, meropenemvaborbactam was associated with higher rates of clinical cure than BAT at both EOT [65.6\% (21/ 32 ) vs. $33.3 \%$ (5/15); difference, $32.3 \%$; $95 \% \mathrm{CI}$ $3.3-61.3 \%, P=0.03]$ and TOC $[59.4 \%(19 / 32)$ vs. $26.7 \%(4 / 15)$; difference, $32.7 \%$; $95 \%$ CI $4.6-60.8 \% ; P=0.02$ ] (Table 2 ). Primary reasons for clinical failure in both groups were death and discontinuation of study drug (due to either death, clinical failure/need for additional antimicrobials, or AEs). Only 1 patient in the meropenem-vaborbactam group within mCREMITT required adjudication of clinical outcome by the blinded committee, representing high concordance between blinded and unblinded assessments. No patients in the BAT mCREMITT group required adjudication due to discordance. Day-28 all-cause mortality was numerically lower with meropenem-vaborbactam versus BAT $[15.6 \%(5 / 32)$ vs. $33.3 \%(5 / 15)$; difference, $-17.7 \%$; $95 \%$ CI -44.7 to $9.3 \%$; $P=0.20]$. While 1 in 5 deaths in the meropenem-vaborbactam group was associated with an $\mathrm{AE}$ term of sepsis, 4 of the 5 deaths in the BAT group were associated with AE terms of sepsis/septic shock (eTable 6). A trend towards significantly greater microbiologic cure was found at EOT in the meropenem-vaborbactam group compared to the BAT group [65.6\% (21/ $32)$ vs. $40.0 \%$ (6/15); difference, $25.6 \%$; $95 \% \mathrm{CI}$ -4.1 to $55.4 \%$; $P=0.09]$; at TOC, this difference was $19.8 \%(53.1 \%$ [17/32] vs. $33.3 \%$ [5/15]; 95\%CI, -9.7 to $49.3 \% ; P=0.19$ ) (Table 2 ).
Within infection type categories in the mCRE-MITT population, Day-28 all-cause mortality rates were numerically lower in patients with HABP/VABP or bacteremia who received meropenem-vaborbactam than in those who received BAT $[22.2 \%(4 / 18)$ vs. $44.4 \%(4 / 9)$, difference, $-22.2 \%$; $95 \%$ CI -59.9 to $15.5 \%$; $P=0.25$ )] (eTable 7). In patients with cUTI/AP, overall success rates at EOT were numerically higher among patients who received meropenem-vaborbactam than those who received BAT $[75.0 \%(9 / 12)$ vs. $50.0 \%(2 / 4)]$; overall success rates at TOC were $33.3 \%(4 / 12)$ for meropenem-vaborbactam and 50.0\% (2/4) for BAT. Among the few patients with cIAI, the clinical cure rate at TOC was $100 \%(2 / 2)$ in the meropenem-vaborbactam group and $0 \%(0 / 2)$ in the BAT group. Efficacy endpoints in patients with all infection types in the MITT and m-MITT populations are shown in eTables 8 and 9 , respectively.

In view of the imbalance in the proportion of randomized patients with prior antimicrobial failure, a sensitivity analysis was performed. Six of 8 clinical failures and 4 of 5 on meropenem-vaborbactam (mCRE-MITT) occurred in patients with prior antibiotic failure. In a sensitivity analysis across infection types, an increase in the treatment effect of meropenem-vaborbactam over BAT for both clinical cure at TOC $[69.6 \%(16 / 23)$ vs. $26.7 \%$ (4/15); 95\% CI of difference, 13.7-72.1\%; $P=0.004]$ and all-cause mortality $[4.3 \%(1 / 23)$ vs. .33.3\% (5/15); $95 \%$ CI of difference, -54.3 to $-3.7 \% ; P=0.02]$ was seen in patients without prior antibiotic failure (Table 2). Additional subgroup analyses showed consistent improvement in clinical cure and decreased mortality associated with meropenem-vaborbactam (Fig. 2). Among immunocompromised patients specifically, meropenem-vaborbactam showed substantially higher cure rates than BAT at TOC [63.6\% (7/11) vs. $0.0 \%(0 / 8) ; 95 \%$ CI of difference, 35.2-92.1\%; $P<0.001]$.

\section{Safety and Tolerability}

In the safety population (same as the MITT population), meropenem-vaborbactam was 


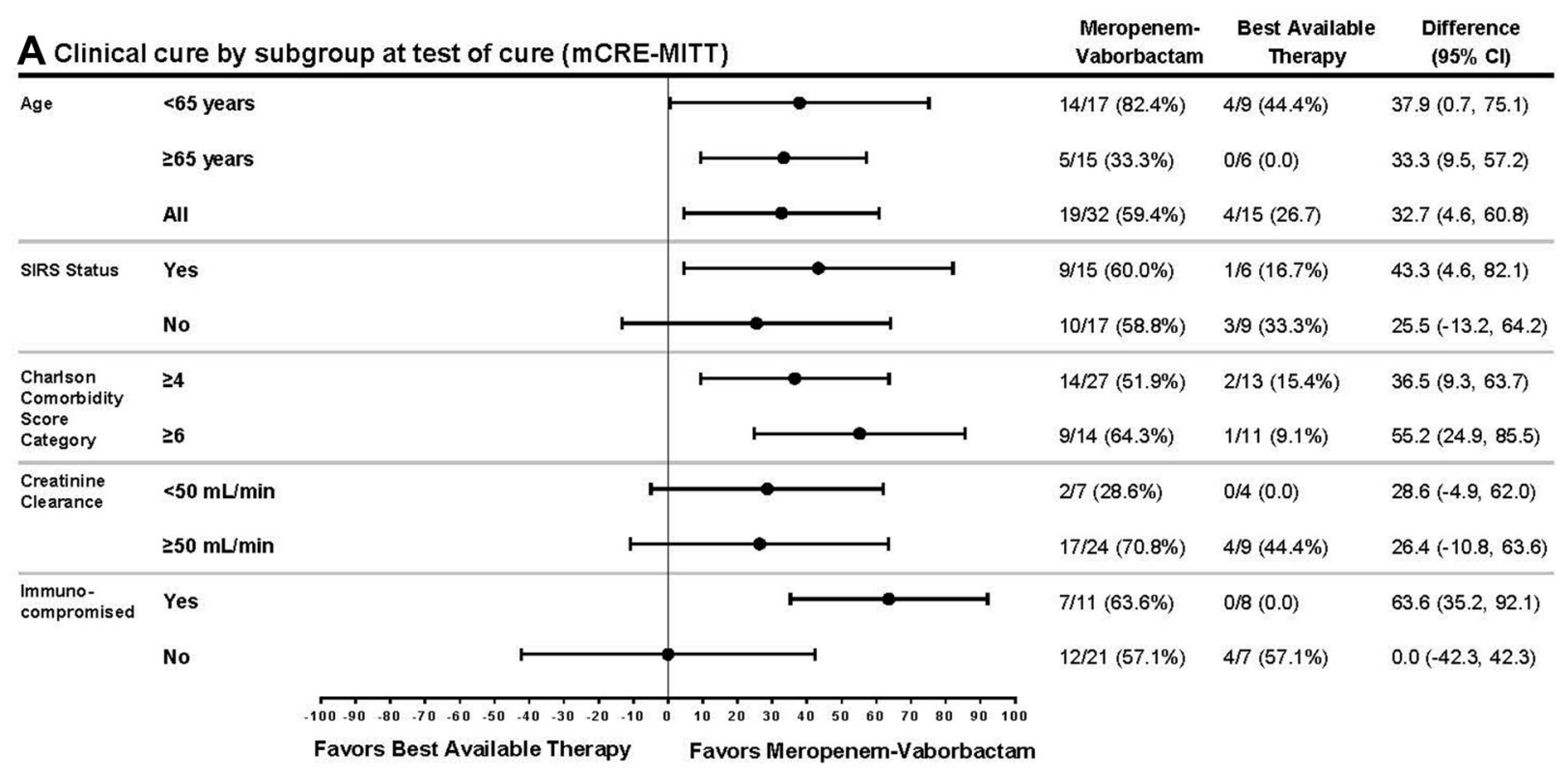

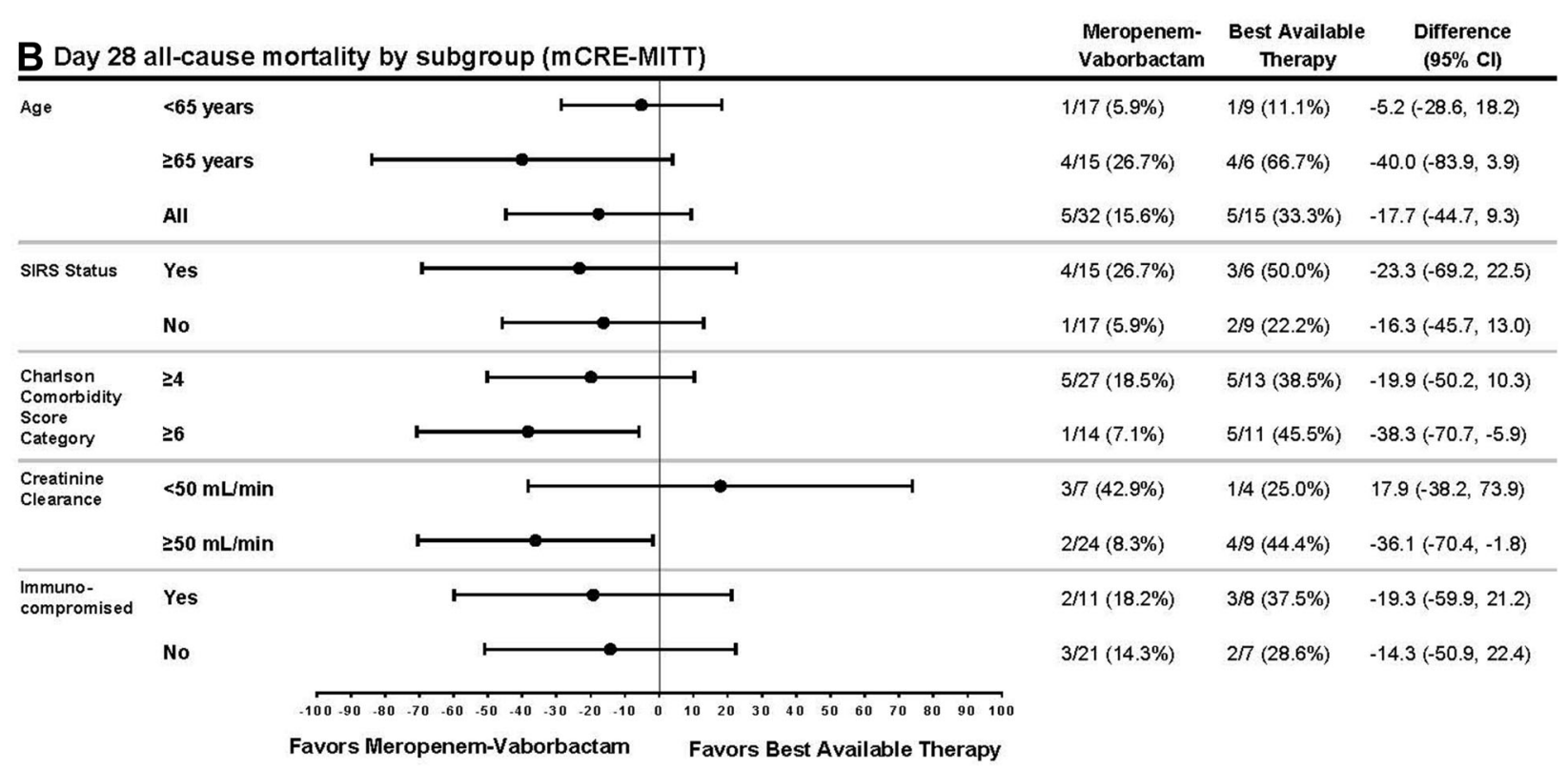

Fig. 2 Subgroup analysis. $m C R E-M I T T$ microbiologic carbapenem-resistant Enterobacteriaceae modified intent-to-treat, SIRS systemic inflammatory response syndrome

associated with fewer TEAEs [84.0\% (42/50) vs. $92.0 \%(23 / 25)]$, severe TEAEs [14.0\% (7/50) vs. $28.0 \%(7 / 25)]$, drug-related TEAEs $[24.0 \%(12 /$ $50)$ vs. $44.0 \%(11 / 25)]$, and serious AEs $[34.0 \%$ $(17 / 50)$ vs. $44.0 \%(11 / 25)]$ than BAT (Table 3$)$. Treatment-related AEs occurring in $>10 \%$ of meropenem-vaborbactam-treated patients included diarrhea, anemia, and hypokalemia. Treatment-related AEs occurring in $>10 \%$ BATtreated patients included sepsis, septic shock, diarrhea, anemia, hypotension, and acute renal failure.

Meropenem-vaborbactam was associated with fewer AEs and laboratory values indicating 
Table 3 Adverse events and safety endpoints (safety population)

\begin{tabular}{|c|c|c|c|}
\hline Adverse events & $\begin{array}{l}\mathrm{M}-\mathrm{V}(n=50) \\
n(\%)\end{array}$ & $\begin{array}{l}\text { BAT }(n=25) \\
n(\%)\end{array}$ & $\begin{array}{l}\text { Total }(N=75) \\
n(\%)\end{array}$ \\
\hline \multicolumn{4}{|l|}{ TEAEs } \\
\hline Any ${ }^{a}$ & $42(84.0)$ & $23(92.0)$ & $65(86.7)$ \\
\hline Diarrhea & $6(12.0)$ & $4(16.0)$ & $10(13.3)$ \\
\hline Anemia & $5(10.0)$ & $3(12.0)$ & $8(10.7)$ \\
\hline Hypokalemia & $5(10.0)$ & $2(8.0)$ & $7(9.3)$ \\
\hline Hypotension & $4(8.0)$ & $3(12.0)$ & $7(9.3)$ \\
\hline Sepsis & $2(4.0)$ & $5(20.0)$ & $7(9.3)$ \\
\hline Septic shock & $1(2.0)$ & $4(16.0)$ & $5(6.7)$ \\
\hline Renal failure acute & $1(2.0)$ & $4(16.0)$ & $4(5.3)$ \\
\hline Drug-related & $12(24.0)$ & $11(44.0)$ & $23(30.7)$ \\
\hline \multicolumn{4}{|l|}{ TEAEs by maximum severity } \\
\hline Mild & $11(22.0)$ & $4(16.0)$ & $15(20.0)$ \\
\hline Moderate & $11(22.0)$ & $5(20.0)$ & $16(21.3)$ \\
\hline Severe & $7(14.0)$ & $7(28.0)$ & $14(18.7)$ \\
\hline Life-threatening & $3(6.0)$ & $1(4.0)$ & $4(5.3)$ \\
\hline Death & $10(20.0)$ & $6(24.0)$ & $16(21.3)$ \\
\hline \multicolumn{4}{|l|}{ SAEs } \\
\hline All & $17(34.0)$ & $11(44.0)$ & $28(37.3)$ \\
\hline Drug-related & $0(0)$ & $2(8.0)$ & $2(2.7)$ \\
\hline Study drug discontinuations due to TEAEs & $5(10.0)$ & $3(12.0)$ & $8(10.7)$ \\
\hline Study discontinuations due to TEAEs & $8(16.0)$ & $5(20.0)$ & $13(17.3)$ \\
\hline \multicolumn{4}{|l|}{ Renal-related safety endpoints } \\
\hline Renal-related TEAEs (Preferred Term) & $2(4.0)$ & $6(24.0)$ & $8(10.7)$ \\
\hline Renal failure acute & $1(2.0)$ & $3(12.0)$ & $4(5.3)$ \\
\hline Renal impairment & $1(2.0)$ & $2(8.0)$ & $3(4.0)$ \\
\hline Renal failure & $0(0)$ & $1(4.0)$ & $1(1.3)$ \\
\hline Any post-baseline RIFLE Criteria ${ }^{b}$ & $1(2.1)$ & $2(8.3)$ & $3(4.2)$ \\
\hline \multicolumn{4}{|l|}{ Maximum post-baseline creatinine increase } \\
\hline$\geq 0.5 \mathrm{mg} / \mathrm{dL}$ & $7(14.0)$ & $6(24.0)$ & $13(17.3)$ \\
\hline
\end{tabular}

$A E$ adverse event, $B A T$ best available therapy, $M-V$ meropenem-vaborbactam, RIFLE risk, injury, failure, loss, or end-stage, $S A E$ serious adverse event, TEAE treatment-emergent adverse event

a TEAEs occurring in a frequency of $\geq 10 \%$ in either treatment arm

b Values at baseline or some post-baseline visit were missing for $2 \mathrm{M}-\mathrm{V}$ patients and 1 BAT patient 
nephrotoxicity versus BAT. A lower incidence of post-baseline increases in serum creatinine of $\geq 0.5 \mathrm{mg} / \mathrm{dL}[14.0 \%(7 / 50)$ vs. $24.0 \%(6 / 25)]$ and renal impairment according to RIFLE criteria $[2.1 \%(1 / 48)$ vs. $8.3 \%(2 / 24)]$, as well as fewer renal-related AEs $[4.0 \%(2 / 50)$ vs. $24.0 \%(6 / 25)]$ were seen with meropenem-vaborbactam than BAT (Table 3).

In light of the combined improvement in efficacy outcomes and reduced renal AEs, exploratory analyses were performed to quantify the risk-benefit profile of meropenem-vaborbactam versus BAT. The composite endpoint of Day-28 all-cause mortality or nephrotoxicity (defined as either $\geq 1.0 \mathrm{mg} / \mathrm{dL}$ increase in postbaseline creatinine or renal AEs) demonstrated improvement in the risk-benefit profile with meropenem-vaborbactam compared to BAT (25.0\% vs. $40.0 \%$; $95 \%$ CI of difference, $-44.0 \%$ to $14.0 \% ; P=0.31$ and $18.8 \%$ vs. $60.0 \%$; $95 \% \mathrm{CI}$ -69.5 to $-13.0 \%$; $P=0.004$, by each nephrotoxicity definition, respectively). Composite endpoints of clinical failure or nephrotoxicity and clinical failure or renal AEs were also associated with a superior risk-benefit profile for meropenem-vaborbactam compared to BAT (31.3\% vs. $80.0 \%$; $95 \%$ CI -74.6 to $-22.9 \%$, $P<0.001$ and $28.1 \%$ vs. $80.0 \% ; 95 \% \mathrm{CI}-77.4$ to $-26.3 \%, P<0.001$ ) (Table 2).

\section{DISCUSSION}

TANGO II is the first prospective, Phase 3 comparative trial of monotherapy with a novel agent in patients with CRE infections. While development of antimicrobials targeting Gramnegative pathogens has increased in recent years, few have been evaluated prospectively in patients with CRE infections. The TANGO II population represented patients with CRE infections [26, 27], including patients with multiple comorbidities, underlying immunocompromise, and moderate-to-severe renal impairment. Importantly, meropenem-vaborbactam was given as monotherapy. In contrast, the comparator, "best-available therapy", reflected usual care for CRE-an individualized cocktail of antibiotics from different classes (e.g., tetracyclines, carbapenems, polymyxins, and aminoglycosides), which often individually have poor activity against CRE and increased toxicities, particularly nephrotoxicity. Consistent with prior retrospective series, BAT in this trial included combination regimens, often with a high-dose carbapenem [12, 26, 27].

The most frequent primary pathogen in this study, KPC-producing K. pneumoniae (in the mCRE-MITT population), $72.7 \%$ of $K$. pneumoniae were KPC-producing, reflects CRE epidemiology in the United States and most regions of the world $[28,29]$. KPC-producing $K$. pneumoniae MIC values for meropenem in this trial $(64 \mu \mathrm{g} / \mathrm{mL})$ demonstrate the high degree of resistance among CRE isolates studied.

The trial showed reduced all-cause mortality, accompanied by a statistically significant increase in cure rates, associated with meropenem-vaborbactam. This increased cure rate was demonstrated even in immunocompromised patients, a group typically excluded from clinical trials, yet at high risk for CRE infections. Meropenem-vaborbactam was also associated with decreased mortality in patients with $\mathrm{HABP} / \mathrm{VABP}$ or bacteremia, the most serious CRE infections. Inclusion of these high-risk populations likely contributed to the ability to detect clinically meaningful differences between meropenem-vaborbactam and BAT.

Nephrotoxicity management is challenging in patients with serious Gram-negative infections, particularly in vulnerable populations (critically ill, immunocompromised) [16, 30]. Patients randomized to meropenem-vaborbactam experienced fewer AEs and decreased nephrotoxicity versus BAT. Together, higher cure rates and lower mortality and nephrotoxicity rates with meropenem-vaborbactam versus BAT prompted the DSMB to recommend ending randomization to BAT following their review during an interim analysis.

Limitations of TANGO II include small sample size, despite being the largest trial to date specifically for CRE. The study was open-label to enable investigator selection and maintenance of BAT. This limitation was mitigated by investigator requirement to define BAT prior to randomization and incorporation of both a blinded local site evaluation and a separate blinded adjudication committee to assess outcomes. The 
low number of cases requiring adjudication and directional consistency of improved cure rates along with reduced mortality suggests that this limitation should not confound trial conclusions.

\section{CONCLUSION}

In summary, treatment of serious CRE infections with meropenem-vaborbactam monotherapy was associated with clinically significant improvement in clinical cure rates, lower nephrotoxicity rates, and marked trend towards lower mortality than BAT. Meropenem-vaborbactam is a valuable new addition to the antimicrobial armamentarium against CRE pathogens.

\section{ACKNOWLEDGEMENTS}

Funding. This project has been funded in part by The Medicines Company and the Department of Health and Human Services; Office of the Assistant Secretary for Preparedness and Response; Biomedical Advanced Research and Development Authority (BARDA), under Contract No. HHSO100201400002C with Rempex Pharmaceuticals, a wholly-owned subsidiary of The Medicines Company and Agreement no. HHSO100201600026C with The Medicines Company. The funder of this study was responsible for study design and data collection. The funder was also responsible for the funding of the journal's page processing charges. All coauthors were responsible for data interpretation and writing of this report. All authors had full access to all of the data in this study and take complete responsibility for the integrity of the data and accuracy of the data analysis.

Authorship. All named authors meet the International Committee of Medical Journal Editors (ICMJE) criteria for authorship for this article, take responsibility for the integrity of the work as a whole, and have given their approval for this version to be published.
Disclosures. Richard G. Wunderink received consulting fees from The Medicines Company, Evangelos J. Giamarellos-Bourboulis received grants from Abbott $\mathrm{CH}$, Astellas Pharma Europe, Axis Shield, BioMerieux france, Xbiotech, FrameWork 7 program HemoSpec, Horizon2020 Marie-Curie project European Sepsis Academy, InflaRx GmbH Germany, and The Medicines Company, and has served as a consultant to AbbiVie USA, Biotest Germany, InflaRx GmbH Germany, The Medicines Company,XBiotech, and Abbott $\mathrm{CH}$ the honorarium for these was paid to the University of Athens, honorarium speaker fees were paid to the University of Athens by Brahms GmbH., Galia Rahav received grants and consulting fees from MSD and Pfizer, and received lecture fees from Pfizer and Astellas, Amy J. Mathers received grants and consulting fees from The Medicines Company, Matteo Bassetti received lecture and/or consultancy fees from MSD, Pfizer, and Astellas, and received grants from MSD and Pfizer, Oliver A. Cornely received grants from Actelion, Arsanis, Astellas, AstraZenenca,Basilea, Bayer, Cidara, F2G, Gilead, GSK, Leeds University, The Medicines Company, Medpace, Melinta Therapeutics, Merck/MSD, Miltenyl, Pfizer, Rempex, Roche, Sanofi Pasteur, Scynexis, Seres Therapeutics, and has received consultancy fees from Actelion, Amplyx, Astellas, Basilea, Cidara, Da Volterra, F2G, Gilead, Janssen Pharmaceuticals, Matinas, Menarini Ricerche, Merck/MSD, Paratek Pharmaceuticals, Pfizer, PSI, Scynexis, Seres Therapeutics, Summit, Tetraphase, Vical, IQVIA, and has served on advisory boards for Da Volterra and F2G, Gilead, Merck/MSD, Paratek Pharmaceuticals, and Summit. Joseph Solomkin received consultancy fees from Rempex and The Medicines Company. George L. Daikos received grants from Pfizer, MSD and Gilead, and received consultancy fees from Pfizer, Achaogen, MSD and Gilead. Tim Felton received consultancy fees from Gilead, GSK and Pfizer. Elizabeth L. Alexander was an employee of The Medicines Company during the study and retains stock on the Medicines Company. Elizabeth L. Alexander's current affiliation is Nabriva Therapeutics, King of Prussia, PA, USA. David Griffith was employed by The Medicines Company during the study received fees for a US 
Government contract HHSO100201400002C, under the Department of Health and Human Services; Office of the Assistant Secretary for Preparedness and Response; Biomedical Advanced Research and Development Authority (BARDA)., Olga Lomovskaya was employed by The Medicines Company during the study, and holds the patent which covers Vaborbactam; 9296763; Patent: Cyclic boronic acid ester derivatives and therapeutic uses thereof. Jeffery Loutit was an employee and shareholder at The Medicines Company. Michael N. Dudley was employee and shareholder of The Medicines Company. Shu Zhang was an employee of The Medicines Company at the time of the study. Keith S. Kaye received consulting fees from Melinta Therapeutics, Allergan, Merck, and Shinogi, and received grants from Merck. The following authors have nothing to disclose: $\mathrm{J}$. Vazquez, T. Bhowmick, J. Bishara, M. Jose Lopez Furst, E. Jeong Kwak, F. Menichetti, I. Oren.

Additional Contributions. We thank the TANGO II investigators who made this study possible. A full list of TANGO II investigators is provided in the Supplemental Material. We thank Dan White, Elizabeth Morgan, Brian Murphy, and Carrie Messerschmidt for thoughtful contribution to this work. Medical writing and editorial support was provided by Purvi Kobawala Smith of Health and Wellness Partners, Upper Saddle River, NJ through funding by The Medicines Company.

Prior Presentation. These data were previously presented, in part, in abstract and poster form at the Annual Meeting of IDSA, October 2017, San Diego CA, USA; European Society of Clinical Microbiology and Infectious Diseases, April 21-24, Madrid Spain; American Society of Microbiology, June 7-11 Atlanta, GA, USA.

Compliance with Ethics Guidelines. Protocol and informed consent form were approved by the site's Institutional Review Board/Independent Ethics Committee. The trial was conducted in accordance with the International Conference on Harmonisation Good Clinical Practice Guideline and with the 1964 Helsinki declaration and its later amendments or comparable ethical standards. Prior to initiation of study-related procedures, an informed consent form was signed by the patient or guardian/legal representative.

Data Availability. Data sharing is not applicable to this article as it is proprietary.

Open Access. This article is distributed under the terms of the Creative Commons Attribution-NonCommercial 4.0 International License (http://creativecommons.org/licenses/ by-nc/4.0/), which permits any noncommercial use, distribution, and reproduction in any medium, provided you give appropriate credit to the original author(s) and the source, provide a link to the Creative Commons license, and indicate if changes were made.

\section{REFERENCES}

1. Bassetti M, Poulakou G, Ruppe E, Bouza E, Van Hal SJ, Brink A. Antimicrobial resistance in the next 30 years, humankind, bugs and drugs: a visionary approach. Intensive Care Med. 2017. https://doi. org/10.1007/s00134-017-4878.

2. Centers for Disease Control and Prevention. Antibiotic resistance threats in the United States, 2013. https://www.cdc.gov/drugresistance/threatreport-2013/index.html. Accessed 27 Aug 2017.

3. World Health Organization. Global priority list of antibiotic-resistant bacteria to guide research, discovery, and development of new antibiotics. http:// www.who.int/medicines/publications/global-priori ty-list-antibiotic-resistant-bacteria/en/. Accessed 5 Sep 2017.

4. European Commission. A European One Health action plan against antimicrobial resistance (AMR). https://ec.europa.eu/health/amr/action_eu_ en. Accessed 5 Sep 2017.

5. The Pew Charitable Trusts. GAIN: how a new law is stimulating the development of antibiotics. http:// www.pewtrusts.org/en/research-and-analysis/issuebriefs/2013/11/07/gain-how-a-new-law-is-stimula ting-the-development-of-antibiotics. Accessed 5 Sep 2017.

6. O'Neill J. Tackling drug-resistant infections globally: an overview of our work. The review on antimicrobial resistance. London: Wellcome Trust; 2016. 
7. Pitout JD, Laupland KB. Extended-spectrum betalactamase-producing Enterobacteriaceae: an emerging public-health concern. Lancet Infect Dis. 2008;8(3):159-66.

8. Munoz-Price LS, Poirel L, Bonomo RA, et al. Clinical epidemiology of the global expansion of Klebsiella pneumoniae carbapenemases. Lancet Infect Dis. 2013;13(9):785-96.

9. Giacobbe DR, Del Bono V, Trecarichi EM, et al; ISGRI-SITA (Italian Study Group on Resistant Infections of the Società Italiana Terapia Antinfettiva). Risk factors for bloodstream infections due to colistin-resistant KPC-producing Klebsiella pneumoniae: results from a multicenter case-controlcontrol study. Clin Microbiol Infect. 2015;21(12):1106.e1-1106.e8.

10. Monaco M, Giani $\mathrm{T}$, Raffone $\mathrm{M}$, et al. Colistin resistance superimposed to endemic carbapenemresistant Klebsiella pneumoniae: a rapidly evolving problem in Italy, November 2013 to April 2014. Euro Surveill. 2014;19(42):14-8.

11. Shields RK, Chen L, Cheng S, et al Emergence of ceftazidime-avibactam resistance due to plasmidborne blaKPC-3 mutations during treatment of carbapenem-resistant Klebsiella pneumoniae infections. Antimicrob Agents Chemother. 2017;61(3):3e02097-16-3e020916.

12. Alexander EL, Loutit J, Tumbarello M, et al. Carbapenem-resistant Enterobacteriaceae infections: results from a retrospective series and implications for the design of prospective clinical trials. Open Forum Inf Dis. 2017;4(2):ofx063.

13. Falagas ME, Lourida P, Poulikakos P, Rafailidis PI, Tansarli GS. Antibiotic treatment of infections due to carbapenem-resistant Enterobacteriaceae: systematic evaluation of the available evidence. Antimicrob Agents Chemother. 2014;58(2):654-63.

14. Tumbarello M, Viale P, Viscoli C, et al. Predictors of mortality in bloodstream infections caused by Klebsiella pneumoniae carbapenemase-producing $K$. pneumoniae: importance of combination therapy. Clin Infect Dis. 2012;55(7):943-50.

15. Daikos GL, Tsaousi S, Tzouvelekis LS, et al. Carbapenemase-producing Klebsiella pneumoniae bloodstream infections: lowering mortality by antibiotic combination schemes and the role of carbapenems. Antimicrob Agents Chemother. 2014;58(4):2322-8.

16. Pouch SM, Satlin MJ. Carbapenem-resistant Enterobacteriaceae in special populations: solid organ transplant recipients, stem cell transplant recipients, and patients with hematologic malignancies. Virulence. 2017;8(4):391-402.
17. Hecker SJ, Reddy KR, Totrov M, et al. Discovery of a cyclic boronic acid beta-lactamase inhibitor (RPX7009) with utility vs. class A serine carbapenemases. J Med Chem. 2015;58(9):3682-92.

18. Rubino CM, Bhavnani SM, Loutit JS, Morgan EE, White D, Dudley MN, Griffith DC (2018) Phase 1 study of the safety, tolerability, and pharmacokinetics of vaborbactam and meropenem alone and in combination following single and multiple doses in healthy adult subjects. Antimicrob Agents Chemother. https://doi.org/10.1128/AAC.0222817.

19. Kaye KS, Bhowmick T, Metallidis S, et al. Effect of meropenem-vaborbactam vs. piperacillin-tazobactam on clinical cure or improvement and microbial eradication in complicated urinary tract infection: the TANGO I randomized clinical trial. JAMA. 2018;319(8):788-99.

20. FDA approves new antibacterial drug [press release]. Silver Spring, MD: US Food and Drug Administration; August 29, 2017. https://www.fda.gov/ NewsEvents/Newsroom/PressAnnouncements/ucm 573955.htm. Accessed 3 Sep 2017.

21. Knaus WA, Draper EA, Wagner DP, Zimmerman JE. APACHE II: a severity of disease classification system. Crit Care Med. 1985;13(10):818-29.

22. Clinical and Laboratory Standards Institute. Performance standards for antimicrobial susceptibility testing. Wayne: Clinical and Laboratory Standards Institute; 2014.

23. European Committee on Antimicrobial Susceptibility Testing. Antimicrobial susceptibility testing. 2014. http://www.eucast.org/ast_of_bacteria/. Accessed 27 Aug 2017.

24. US Department of Health and Human Services. Common terminology criteria for adverse events (CTCAE). https://ctep.cancer.gov/protocoldevelop ment/electronic_applications/ctc.htm. Accessed 27 Aug 2017.

25. Bellomo R, Kellum J, Ronco C. Acute renal failure: time for consensus. Intensive Care Med. 2001;27(11):1685-8.

26. Tumbarello M, Trecarichi EM, De Rosa FG, et al. ISGRI-SITA (Italian Study Group on Resistant Infections of the Società Italiana Terapia Antinfettiva). Infections caused by KPC-producing Klebsiella pneumoniae: differences in therapy and mortality in a multicentre study. J Antimicrob Chemother. 2015;70(7):2133-43.

27. Daikos GL, Petrikkos P, Psichogiou $M$, et al. Prospective observational study of the impact of VIM-1 metallo-beta-lactamase on the outcome of 
patients with Klebsiella pneumoniae bloodstream infections. Antimicrob Agents Chemother. 2009;53(5):1868-73.

28. Cerqueira GC, Earl AM, Ernst CM, et al. Multi-institute analysis of carbapenem resistance reveals remarkable diversity, unexplained mechanisms, and limited clonal outbreaks. Proc Natl Acad Sci USA. $2017 ; 114(5): 1135-40$.
29. Glasner C, Albiger B, Buist G, et al Carbapenemaseproducing Enterobacteriaceae in Europe: a survey among national experts from 39 countries, February 2013. Euro Surveill. 2013;18(28):20525. http:// www.eurosurveillance.org/ViewArticle.aspx?Article Id=20525. Accessed 27 Aug 2017.

30. Lewis SJ, Mueller BA. Antibiotic dosing in patients with acute kidney injury: 'enough but not too much'. J Intensive Care Med. 2014;31(3):164-76. 